\title{
聚(L-谷氨酸)微球的制备及其口服药物控释研究
}

\author{
赵丽 $b$ \\ 丁建勋 ${ }^{a}$ \\ 肖春生 $*, a$ \\ 陈学思 $a$ \\ 盖广清 ${ }^{b}$ 王立艳 ${ }^{c}$ \\ ( ${ }^{a}$ 中国科学院长春应用化学研究所 中国科学院生态环境高分子材料重点实验室 长春 130022) \\ ( ${ }^{b}$ 吉林建筑大学 建筑节能技术工程实验室 长春 130118) \\ ( ${ }^{c}$ 吉林建筑大学 材料科学与工程学院 长春 130118)
}

\begin{abstract}
摘要 通过正己胺引发 $\gamma$-苯甲基- $L$-谷氨酸酯- $N$-内羧酸酐(BLG-NCA)开环聚合制备聚( $\gamma$-苯甲基- $L$-谷氨酸酯)(PBLG), 并进一步脱掉苯甲基保护得到聚( $L$-谷氨酸)(PLG). 以利福平为模型药物, 通过油包油 $(\mathrm{O} / \mathrm{O})$ 无水乳液法制备了 PLG 载 药微球. 扫描电子显微镜检测表明该载药微球具有良好的球形形貌且粒径分布较均一, 粒径大小约为 $9.0 \mu \mathrm{m}$. 体外释 放实验表明该载药微球对利福平的释放具有明显的 $\mathrm{pH}$ 敏感性，在模拟胃液中较少释放利福平，而在模拟肠液中较快并 大量释放利福平, 符合口服药物载体释放性能的要求, 可用于口服药物的定位肠溶性载体. 此外, 噻唑蓝实验表明该 微球具有良好的生物相容性.
\end{abstract}

关键词 肠溶性载体；聚( $L$-谷氨酸)；口服给药；微球；药物传递

\section{Poly(L-glutamic acid) Microsphere: Preparation and Application in Oral Drug Controlled Release}

\author{
Zhao, $\mathrm{Li}^{b}$ \\ Ding, Jianxun ${ }^{a}$ \\ Gai, Guangqing ${ }^{b}$ \\ Xiao, Chunsheng ${ }^{*, a}$ \\ Wang, Liyan ${ }^{c}$ \\ Chen, Xuesi ${ }^{a}$ \\ $\left({ }^{a}\right.$ Key Laboratory of Polymer Ecomaterials, Changchun Institute of Applied Chemistry, Chinese Academy of Sciences, \\ Changchun 130022) \\ ( ${ }^{b}$ Laboratory of Building Energy-Saving Technology Engineering, Jilin Jianzhu University, Changchun 130118) \\ ( ${ }^{c}$ College of Material Science and Engineering, Jilin Jianzhu University, Changchun 130118)
}

\begin{abstract}
Poly( $L$-glutamic acid) (PLG) and its derivatives, which are biodegradable and biocompatible, are one kind of the most widely investigated synthetic polypeptides as biomedical materials benefited from their $\mathrm{pH}$-responsive property $\left(\mathrm{p} K_{\mathrm{a}} \sim 4.1\right)$ and modifiable side carboxyl group. In this work, PLG was synthesized via a two-step procedure. Poly $(\gamma$-benzyl- $L$-glutamate) (PBLG) was first synthesized by the ring-opening polymerization of $\gamma$-benzyl- $L$-glutamate- $N$-carboxyanhydride (BLG-NCA) using $n$-hexylamine as initiator, and then PLG was prepared by the deprotection of benzyl groups in PBLG. PLG microsphere was prepared by the oil-in-oil (O/O) anhydrous emulsion method utilizing $N, N$-dimetylformamide as an inner oil phase and corn oil as an outer oil phase. Rifampicin, a model drug, was loaded into the PLG microsphere, and the drug loading capacity and entrapment efficiency were revealed. The scanning electron microscopy micrograph indicated that the drug-loaded microsphere exhibited spherical morphology with narrow size distribution and average diameter at about $9.0 \mu \mathrm{m}$. To investigate the application of PLG microsphere in oral drug delivery (especially for enteric-coated drugs), the in vitro rifampicin release experiments in simulated gastric or intestinal fluid were performed. In vitro release results revealed that the release of rifampicin from microsphere was highly dependent on $\mathrm{pH}$. In detail, less amount of rifampicin was released in simulated gastric fluid, while the quicker release of rifampicin occurred in simulated intestinal fluid. It was because that the deprotonation of carboxyl groups in PLG caused the loose, expansion, and even disintegration of PLG microsphere in simulated intestinal fluid. Moreover, the morphology of microsphere in simulated gastric fluid was different from that in simulated intestinal fluid, which further confirmed the $\mathrm{pH}$-sensitive property of microsphere. Additionally, in vitro methyl thiazolyl tetrazolium assays demonstrated that the PLG microsphere was biocompatible. Therefore, the biocompatible PLG microsphere with the intelligent $\mathrm{pH}$-triggered drug release should be promising for application in oral drug delivery.
\end{abstract}

Keywords enteric carrier; poly( $L$-glutamic acid); oral administration; microsphere; drug delivery

*E-mail: xiaocs@ciac.ac.cn; Tel.: 0431-8526-2116

Received November 9, 2014; published January 6, 2015.

Project supported by the National Natural Science Foundation of China (Nos. 51403075, 51303174, 51203153, 51390484, 51233004, 51321062) and the Scientific Development Program of Jilin Province (No. 20140520050JH).

项目受国家自然科学基金(Nos. 51403075, 51303174, 51203153, 51390484, 51233004, 51321062)及吉林省科技发展计划基金(No. 20140520050JH)资助. 


\section{1 引言}

药物制剂是临床用药形式, 理想的药物剂型应该实 现药物使用剂量最小、副作用最低且疗效发挥最大. 药 物传递系统制剂按照给药途径, 可分为注射给药和非注 射给药, 其中非注射给药包括口服给药 ${ }^{[1]}$ 、鼻腔给药 ${ }^{[2]}$ 、 肺部给药 ${ }^{[3,4]}$ 、透皮给药 ${ }^{[5,6]}$ 以及眼部给药 ${ }^{[7]}$ 等. 相对于注 射给药, 口服给药是一种非侵入式的给药途径, 以其给 药方便、安全、患者易接受等优点, 成为最常用的也是 目前病人顺从性最好的一种给药方式. 药物口服剂型的 制备并不需要满足无菌、无污染等特殊严格的监管要求. 近年来新的口服给药系统, 如自我乳化药物传递、口服 择时释药系统、胃滞留型控释药物系统等, 得以快速发 展 $^{[8]}$. 虽然口服给药具有上述优势并得到了快速的发展, 但对于胰岛素等蛋白类药物而言, 其生物利用度很低, 达不到临床应用的标准. 这主要是由胰岛素等蛋白类药 物本身的结构以及稳定性决定的. 对于大分子的蛋白类 药物, 它具有较大的分子量和较为复杂的空间结构. 当 其口服时, 由于胃中极酸的环境以及胃肠道中大量蛋白 酶的破坏作用, 易导致蛋白类药物的变性, 从而使蛋白 类药物在到达肠道部位前已经部分或全部失活并大大 降低其生物利用度 ${ }^{[9,10]}$

研究开发特殊的药物载体提高口服定位肠溶性药 物的生物利用度有重要的意义. 聚合物微球是被广泛研 究和应用的一类药物载体 ${ }^{[1]}$. 该微球对药物的包裹相 对紧密且制备工艺简单, 能够大量制备. 常用的聚合物 口服微球多采用 $\mathrm{pH}$ 敏感的肠溶性载体材料, 如聚丙烯 酸类聚合物, 该类聚合物在酸性的胃液中不溶解, 可以 很好地保护药物, 但在偏中性的肠液中可以溶解从而将 所包埋的药物释放出来 ${ }^{[12]}$. 但这类聚合物不可降解, 长 期使用的机体安全性有待考察.

聚氨基酸及其衍生物是一类重要的具有优异的生 物可降解性和生物相容性的合成聚合物, 已被广泛应用 于生物医学研究领域, 如药物控制释放、基因转染、抗 菌材料、组织工程等 ${ }^{[13 \sim 18]}$. 聚 $(L$ - 谷氨酸) $(\operatorname{Poly}(L$-glutamic acid), PLG) 是由天然存在的 $L$-谷氨酸通过酰胺键 链接而成的侧基为羧基的高分子. 侧羧基赋予 PLG 良 好的 $\mathrm{pH}$ 敏感性 ${ }^{[19-22]}$, 是口服药物载体的优异材料.

采用先进的制剂技术有望实现蛋白类药物的口服 传递并保证药物安全地到达肠道部位. 本文通过正己胺 引发 $\gamma$-苯甲基- $L$ - 谷氨酸酯- $N$-内羧酸酐(BLG-NCA)开环 聚合得到聚( $(\gamma$-苯甲基- $L$-谷氨酸酯)(PBLG), 进一步脱掉 苯甲基保护制备 PLG. 采用改进的油包油(O/O)无水乳 液法制备 PLG 微球. 该微球在胃液的酸性环境下由于 羧基的质子化能够很好地保护所担载的药物. 在肠液环 境下由于羧基的去质子化, 聚合物微球溶胀甚至解体而 将所担载的药物快速释放出来, 从而有效保障所担载的 药物安全到达肠道. 本文以利福平为模型药物对载药微 球药物控制释放性能进行了详细的表征. 通过考察利福
平的体外释放实验，证实该聚氨基酸微球具有口服定位 肠溶性药物释放的特性, 可用于胰岛素等肠溶性药物的 口服传递, 为口服药物载体的设计与制备提供理论和实 验基础.

\section{2 结果与讨论}

\subsection{PLG 的制备}

在本文中, 通过两步法制备了生物可降解和生物相 容的 PLG. 首先, 通过正己胺引发 BLG-NCA 开环聚合 制备 PBLG. 然后对 PBLG 脱掉苯甲基保护合成 PLG (图 1).
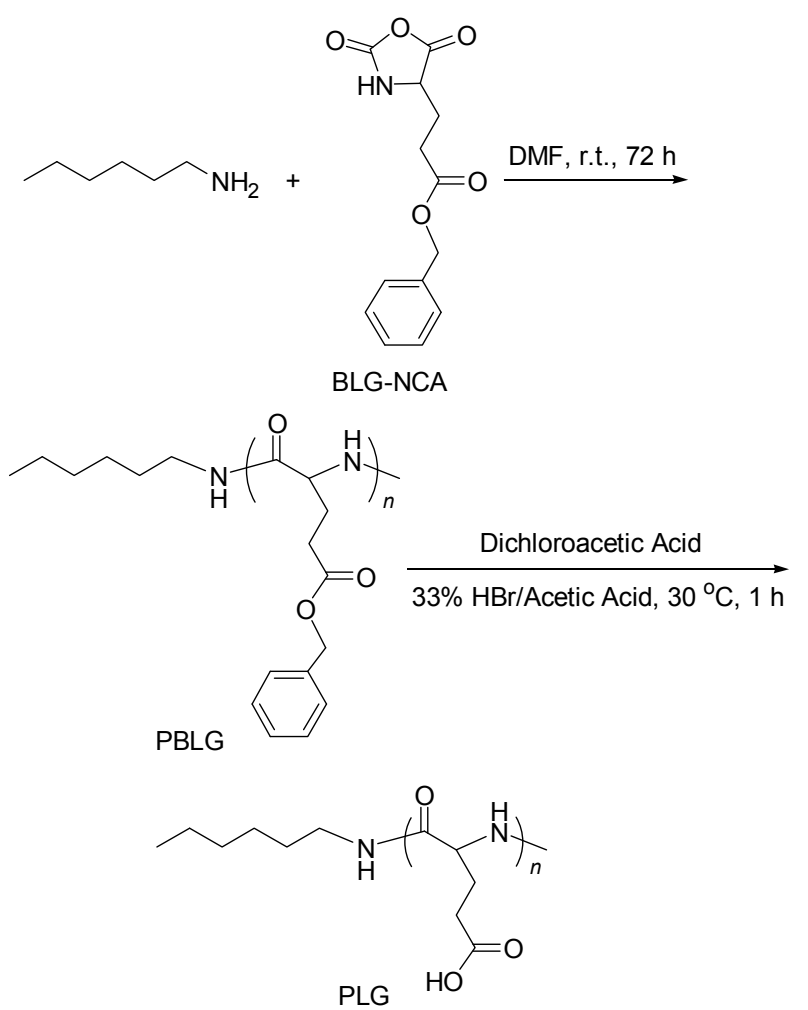

图 1 PLG 的合成路线

Figure 1 Synthetic route of PLG

聚合物的结构通过核磁共振氢谱 $\left({ }^{1} \mathrm{H}\right.$ NMR) 分析得 到证实. 从图 2a 中可以看出, PBLG 的核磁共振特征峰 如下: $\delta 5.12$ 为 $\mathrm{PBLG}$ 链上苯甲基中亚甲基的特征峰 $\left(\mathrm{C}_{6} \mathrm{H}_{5} \mathrm{CH}_{2}-, 2 \mathrm{H}\right) ; \delta 4.67$ 处的共振峰是 PBLG 主链中质子 的特征峰 $\left(-\mathrm{C}(\mathrm{O}) \mathrm{C} \boldsymbol{H}\left(\mathrm{CH}_{2}\right) \mathrm{NH}^{-}, 1 \mathrm{H}\right) ; \delta 2.52 、 2.15$ 和 2.0 处出现的共振信号分别是 PBLG 侧基上的亚甲基的特征 峰 $\left(-\mathrm{C}(\mathrm{O}) \mathrm{CH}_{2} \mathrm{CH}_{2}-, 2 \mathrm{H},-\mathrm{C}(\mathrm{O}) \mathrm{CH}_{2} \mathrm{CH}_{2}-, 1 \mathrm{H}\right.$ 和 $\left.1 \mathrm{H}\right) ; \delta$ 0.86 是正己胺中甲基的特征峰 $\left(\mathrm{CH}_{3} \mathrm{CH}_{2}-, 3 \mathrm{H}\right) ; \delta 1.14 \sim$ 1.52 是正己胺中靠近甲基的四个亚甲基的特征峰 $\left(\mathrm{CH}_{3}\left(\mathrm{CH}_{2}\right)_{4}{ }^{-}, 8 \mathrm{H}\right)$. 通过 $\delta 0.86$ 和 2.52 特征峰的峰面积 之比得知, PBLG 中 BLG 的聚合度为 116. PLG 的制备是 通过 PBLG 脱除苯甲基保护得到的，且其结构同样通过 ${ }^{1} \mathrm{H}$ NMR 得以证实, 如图 $2 \mathrm{~b}$ 所示. 通过对比图 2a 


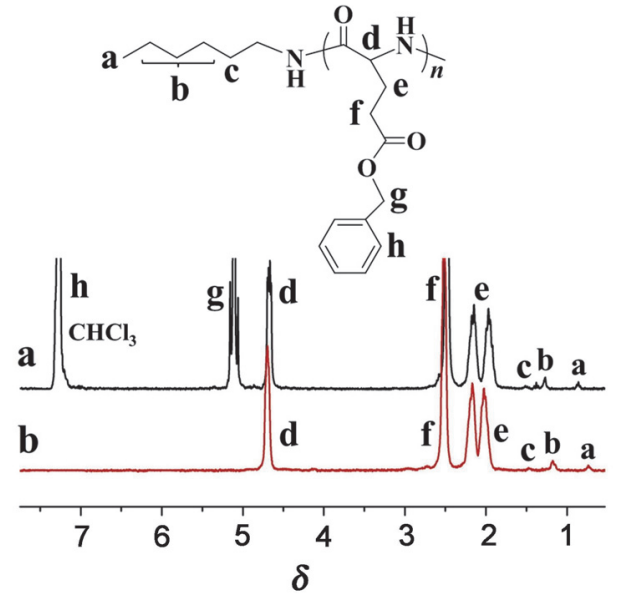

图 2 PBLG (a, TFA- $\left.d / \mathrm{CDCl}_{3}(1 / 1, V / V)\right)$ 和 PLG $(\mathrm{b}, \mathrm{TFA}-d)$ 的 ${ }^{1} \mathrm{H}$ NMR 谱图

Figure $2{ }^{1} \mathrm{H}$ NMR spectra of PBLG (a, TFA- $\left.d / \mathrm{CDCl}_{3}(1 / 1, V / V)\right)$ and PLG (b, TFA- $d$ )

和 $2 \mathrm{~b}$ 可知在 $\delta 7.28$ 和 5.12 处的共振信号峰消失, 证明 苯甲基的成功脱除. 此外, 脱除苯甲基保护前后聚合物 的聚合度并未发生明显变化, 证实脱保护反应并没有造 成聚合物的断链. 凝胶渗透色谱 (GPC) 测试结果也证实 了 PBLG 的成功合成和脱保护反应的发生(表 1). 所制 备的聚合物具有较窄的分子量分布, 且脱除苯甲基保护 后聚合物的分子量分布变窄, 这可能是由于脱保护反应 后经过透析除去了小分子量的 PLG 的缘故.

表 1 聚合物的表征

Table 1 Characterizations of polymers

\begin{tabular}{llll}
\hline Entry & $M_{\mathrm{n}}{ }^{a}$ & $M_{\mathrm{n}}{ }^{b}$ & PDI $^{b}$ \\
\hline PBLG & 25400 & 25400 & 1.81 \\
PLG & 15000 & 15700 & 1.38 \\
\hline
\end{tabular}

${ }^{a}$ Determined by ${ }^{1} \mathrm{H} \mathrm{NMR} ;{ }^{b}$ Determined by GPC.

\section{2 载药 PLG 微球的制备}

采用油包油 $(\mathrm{O} / \mathrm{O})$ 无水乳液的方法制备了 PLG 空白 微球和担载利福平的微球. 制备出来的聚合物空白微球 的形貌如图 3a 所示. 从 SEM 电镜照片中看到, 所制备 的载药聚合物微球具有表面光滑的球形形貌. 微球粒径 在 $9.0 \mu \mathrm{m}$ 左右, 粒径分布相对均一, 且表面光滑. 载药 PLG 微球与空白 PLG 微球形貌相似, 但不如空白微球 表面光滑(图 3b), 这是因为在载药过程中利福平并没有 完全包埋在微球内部所致.

载药 PLG 微球的载药量为 $4.2 \mathrm{wt} \%$, 载药效率为 $46.2 \mathrm{wt} \%$. 微球对于利福平的包封率偏低是由于利福平 是一种脂溶性的药物, 在外相溶剂玉米油中具有一定的 溶解度. 因此, 一部分药物在微球制备过程中溶解在外 相中从而导致载药量和载药效率较低.

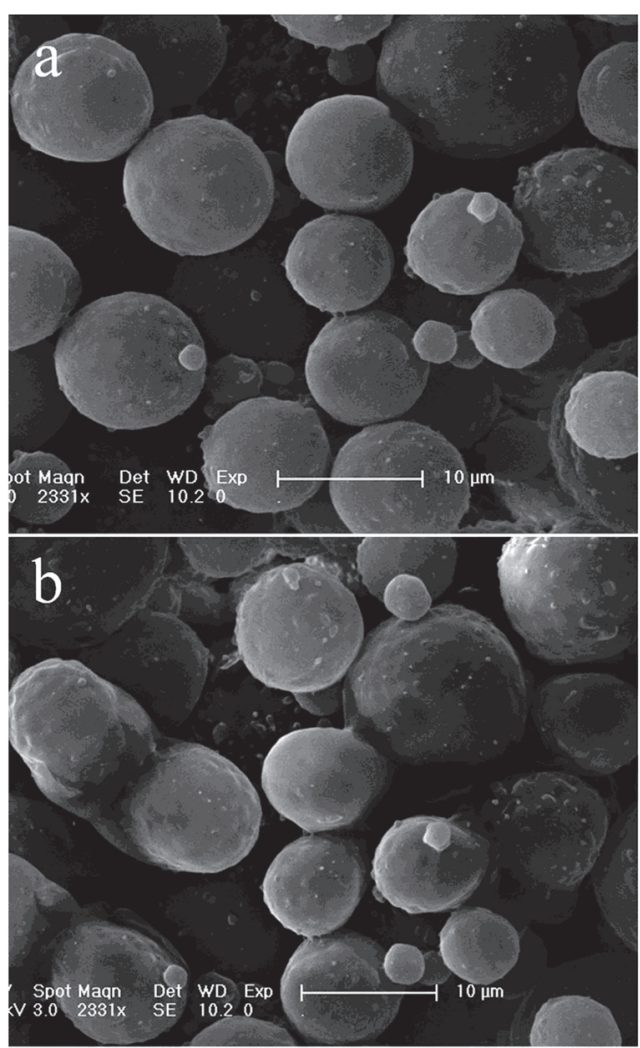

图 3 空白微球(a)和载药微球(b)的 SEM 电镜照片

Figure 3 Typical SEM micrographs of blank (a) and drug-loaded microspheres (b)

\section{3 载药 PLG 微球的体外释放}

考察载药微球分别在模拟胃液的酸性缓冲液中 $(\mathrm{pH}$ $=1.2)$ 和模拟肠液的中性缓冲液中 $(\mathrm{pH}=6.8)$ 的利福平释 放行为, 结果如图 4 所示. 在酸性的无酶的模拟胃液中, 利福平释放较为缓慢, $120 \mathrm{~min}$ 累积释放量为 $17.6 \%$. 而 微球在 $\mathrm{pH}=6.8$ 的不含酶的模拟肠液中, 药物呈持续而 迅速的释放特性，累积释放时间为 $200 \mathrm{~min}$ 时，利福平

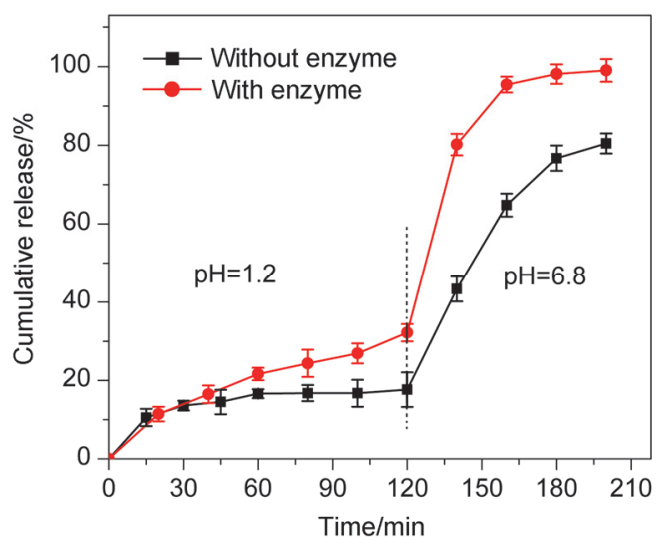

图 4 担载利福平的 PLG 微球在不含或含胃蛋白酶的模拟胃液和不 含或含胰蛋白酶的模拟肠液中的体外释放

Figure 4 In vitro cumulative rifampicin release from rifampicin-loaded PLG microsphere in simulated gastric fluid without or with pepsin, and simulated intestinal fluid without or with trypsin 
的累积释放量为 $80.1 \%$, 符合口服药物肠溶性载体的要 求. 微球在两种不同 $\mathrm{pH}$ 的缓冲液中的药物释放行为完 全不同. 如图 5 所示, 由于 PLG 侧基带有大量的羧基, 微球在酸性环境中由于羧基质子化而不溶解, 致使微球 变得更密实从而有效保护药物. 在模拟胃液中利福平的 释放则是由接近微球表面和内部少量的利福平通过扩 散作用而释放的, 同时由于利福平在水中的溶解度不 大, 因此释放比较缓慢. 在模拟肠液中, PLG 侧链的羧 基去质子化, 材料逐渐溶胀从而使微球疏松膨胀甚至解 体而将所担载的药物快速释放出来.
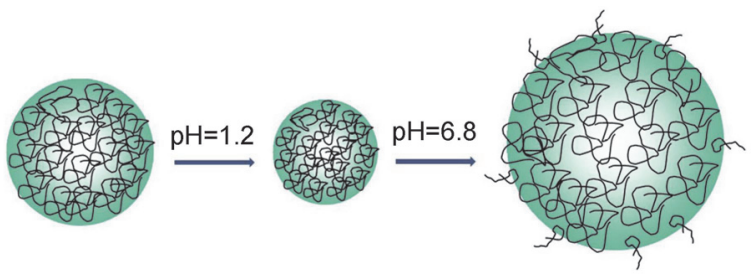

图 5 微球的 $\mathrm{pH}$ 敏感性示意图

Figure 5 Schematic illustration of $\mathrm{pH}$-sensitive property of microsphere

在含有蛋白酶的模拟胃液和模拟肠液中, 利福平的 累积释放仍然具有较明显的 $\mathrm{pH}$ 敏感性. 在含有胃蛋白 酶的模拟胃液中, 利福平的累积释放量增加并不十分明 显, 这是由于在较低 $\mathrm{pH}$ 时 PLG 疏水, 胃蛋白酶对 PLG 的降解作用不明显. 而在含有胰蛋白酶的模拟肠液中, 由于胰蛋白酶的降解作用, 利福平的累积释放量呈现明 显增加的趋势, 更加符合口服药物肠溶性释放特性的要 求 ${ }^{[23]}$.

为进一步验证载药 PLG 微球的 $\mathrm{pH}$ 敏感药物释放特 性, 我们考察了其在模拟胃液和模拟肠液中浸泡之后的 形貌. 如图 $6 \mathrm{a}$ 所示, 微球在 $\mathrm{pH}=1.2$ 的缓冲液中浸泡 $1 \mathrm{~h}$ 后微球的形貌变化不大, 但微球的大小有所减小, 微球 的完整性仍能保持, 说明在模拟胃液的酸性环境下微球 对所担载的药物具有一定的保护作用. 而在中性缓冲液 中浸泡 $0.5 \mathrm{~h}$ 后的微球虽能保持球形, 但微球尺寸有所 变大, 表面出现很多小孔洞且微球表面粗粘(图 6b), 进 一步证实在模拟肠液中微球能够快速响应 $\mathrm{pH}$ 的变化而 将药物快速释放出来. 这也可以侧面说明在中性缓冲液 中药物的释放是由于微球的溶蚀而加速, 该载药 PLG 微球具有口服药物肠溶性释放的特性, 可用于胰岛素等 肠溶性药物的口服传递.

\subsection{PLG 微球的细胞毒性分析}

PLG 作为一种合成的高分子药用辅料, 要在体内得 以应用, 必须确保材料具有良好的生物相容性. 本文中 采用 MTT 法来评价 PLG 对 HeLa 细胞的体外细胞相容 性. 以十二烷基硫酸钠(SDS)作为阳性对照, 考察细胞 与不同浓度的 PLG 材料以及空白 PLG 微球共培养 $72 \mathrm{~h}$ 后的 HeLa 细胞的成活率, 实验结果如图 7 所示. 在实验 浓度高达 $1.0 \mathrm{mg} \cdot \mathrm{mL}^{-1}$ 的浓度范围内, 与 PLG 共培养的 $\mathrm{HeLa}$ 细胞具有 $90 \%$ 以上的生存能力, 表明该 PLG 材料
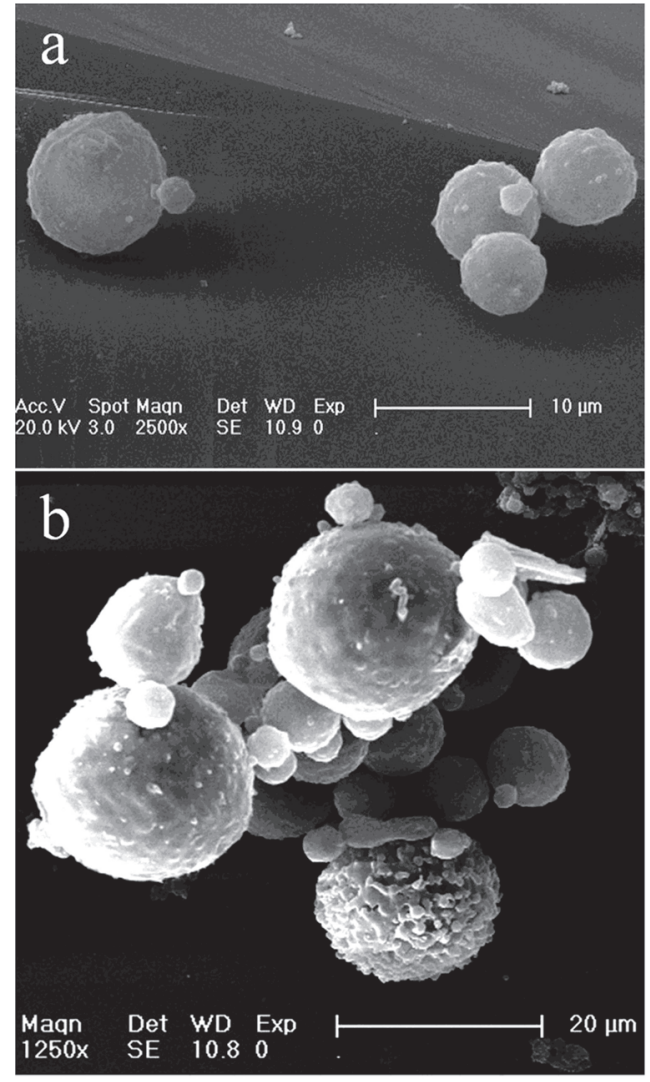

图 6 载药 PLG 微球在模拟胃液(a)和模拟肠液(b)中浸泡后的扫描电 镜照片

Figure 6 Typical SEM micrographs of PLG microspheres immersed in simulated gastric (a) or intestinal fluids (b)

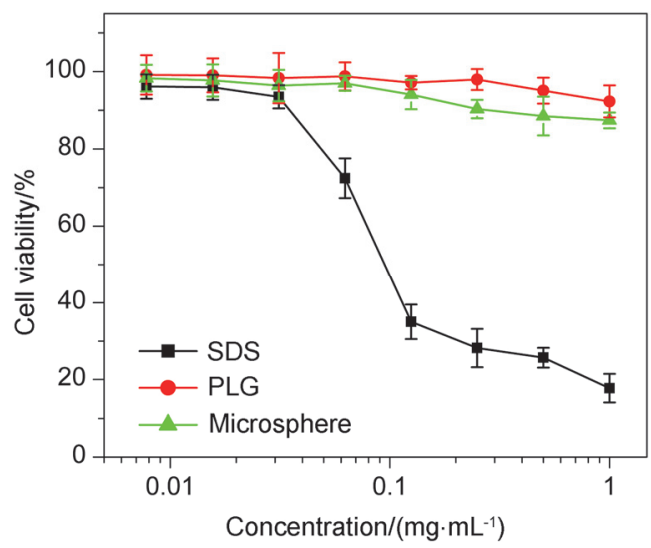

图 7 PLG 和空白 PLG 微球对 HeLa 细胞的细胞毒性, 以 SDS 为阳性 对照. 数据表示为平均值 \pm 标准方差 $(n=6)$

Figure 7 In vitro cytotoxicities of PLG and blank PLG microspheres to HeLa cells with SDS as positive control. Data were expressed as mean \pm standard deviation $(n=6)$

具有较低的毒性和良好的细胞相容性. 同样地, 经过空 白 PLG 微球处理的 HeLa 细胞在微球浓度高达 1.0 $\mathrm{mg} \cdot \mathrm{mL}^{-1}$ 时仍具有 $85 \%$ 以上的存活率. 对比 PLG 材料和 PLG 微球, 细胞毒性没有显著差异, 均能保持较高的细 胞存活率, 表明 PLG 微球的制备工艺是安全可靠的, 并 
没有引入毒性物质，可以应用于口服药物的载体.

\section{3 结论}

本工作采用 BLG-NCA 开环聚合并进一步脱掉苯甲 基保护得到 PLG, 并通过 ${ }^{1} \mathrm{H}$ NMR 对其结构进行分析. 采用 $\mathrm{O} / \mathrm{O}$ 无水乳液法制备了载有利福平的 PLG 微球. SEM 表明该载药微球具有表面光滑的球形形貌. 体外 模拟释放实验表明该微球具有良好的 $\mathrm{pH}$ 响应性, 在模 拟胃液中利福平释放缓慢, 而在模拟肠液中利福平则快 速且持续地释放出来, 符合口服药物载体释放性能的要 求. 载药微球在模拟胃液和模拟肠液中的形貌也进一步 证实了该微球的 $\mathrm{pH}$ 敏感药物释放性能. 此外, MTT 实 验表明 PLG 材料及其空白微球均没有明显的细胞毒性, PLG 微球的制备工艺安全可靠且无毒性物质引入. 因 此, 该生物相容性好的具有 $\mathrm{pH}$ 响应药物释放性能的 PLG 微球有望应用于口服定位肠溶性给药载体.

\section{4 实验部分}

\section{1 试剂与材料表征}

BLG-NCA 的制备依照文献方法 ${ }^{[24]}$. $N, N$-二甲基甲 酰胺(DMF)用前经氢化钙 $\left(\mathrm{CaH}_{2}\right)$ 回流, 并减压蒸馏. 玉 米油为市售产品. 其他所有反应物和溶剂均从国药集团 化学试剂有限公司购得.

用 Bruker AV $400 \mathrm{NMR}$ 核磁共振谱仪测试材料的 ${ }^{1} \mathrm{H}$ NMR. 所用溶剂为気代三氟乙酸 $(\mathrm{TFA}-d)$ 和気代氯仿 $\left(\mathrm{CDCl}_{3}\right)$. 所用内标为 $0.01 \%(V / V)$ 四甲基硅烷(TMS). SEM 测试所用仪器为 Micrion FEI PHILIPS. 为了观察 聚合物微球的表面形态和尺寸, 聚合物微球在测试前进 行喷金处理. 高剪切乳化机为上海威宇机电制造有限公 司, 1001x 型乳化机, 速度 $0 \sim 11000 \mathrm{r} / \mathrm{min}$, 定子直径 35 $\mathrm{mm}$.

\subsection{PLG 的制备}

PLG 的制备依照文献方法 ${ }^{[21]}$, 所不同的是引发剂 为正己胺, 溶剂为无水氯仿. 正己胺引发 BLG-NCA 开 环聚合得到 PBLG, 产率为 89.9\%. PLG 则通过 PBLG 脱 除苯甲基保护基而制得，产率为 $74.2 \%$.

\section{3 载药 PLG 微球的制备}

担载利福平的 PLG 微球采用 $\mathrm{O} / \mathrm{O}$ 的无水乳液方法 制备 ${ }^{[25]}$. 称取 $180.0 \mathrm{mg}$ 的 PLG 和 $18.0 \mathrm{mg}$ 的利福平, 加 入 $12.0 \mathrm{~mL}$ 的 DMF, 搅拌至 PLG 和利福平溶解. 将上述 DMF 溶液注入 $120.0 \mathrm{~mL}$ 含有 $1.2 \mathrm{~g}$ 司班 80 的玉米油中, 高速剪切(4000 r/min)乳化 $5 \mathrm{~min}$, 得到油包油乳液. 乳 化后在搅拌条件下向乳液中滴加 $180.0 \mathrm{~mL}$ 乙醚, 搅拌 2 $\mathrm{h}$, 使内相凝固成为微球. 静置, 待微球沉下后弃去乙醚 收集微球, 并用 $100.0 \mathrm{~mL}$ 乙醚洗涤三次, 风干. 得到包 裹利福平的 PLG 微球粉末.

空白微球的制备与载药微球方法类似, 制备过程中
不加入利福平.

\section{4 载药 PLG 微球的表征}

\subsection{1 微球载药量的测定}

通过碱性 SDS 萃取的方法测定微球中利福平的载 药量 ${ }^{[26]}$. 称取一定量的载药微球, 加入 $2.0 \mathrm{~mL}$ 的 $\mathrm{NaOH}-$ SDS 降解液 $(5.0 \mathrm{wt} \% \mathrm{SDS}, 0.1 \mathrm{~mol} / \mathrm{L} \mathrm{NaOH})$, 置入恒温 振荡箱中振荡, 待微球完全溶解得到均一透明的溶液, 通过紫外分光光度计测定 $334 \mathrm{~nm}$ 处的吸收, 通过标准 曲线测得利福平浓度.

载药量 $(w t \%)=[$ 微球中药物质量 $] /[$ 微球总质量 $] \times 100$

载药效率 $(w \mathrm{t} \%)=[$ 实际载药量 $] /[$ 理论载药量 $] \times 100$

\subsection{2 微球形貌}

微球的形貌通过 SEM 观察. 将微球的悬浊液滴于 硅片上，晾干后表面喷金处理，通过 SEM 观察.

\subsection{3 载药微球在不同 $\mathrm{pH}$ 缓冲液中的释放行为}

配制模拟消化道 $\mathrm{pH}$ 值的缓冲液(简称模拟胃液和模 拟肠液): 取浓盐酸 $7.0 \mathrm{~mL}$, 加水约 $800.0 \mathrm{~mL}$, 并加入 $2.0 \mathrm{~g}$ 的氯化钠, 调节至 $\mathrm{pH}$ 值至 1.2 , 加水稀释至 1000.0 $\mathrm{mL}$, 即得模拟胃液. 取磷酸二氢钾 $6.8 \mathrm{~g}$, 加水 $500 \mathrm{~mL}$ 使溶解, 用 $0.1 \mathrm{~mol} / \mathrm{L}$ 的氢氧化钠溶液调节 $\mathrm{pH}$ 值至 6.8 , 加水稀释至 $1000 \mathrm{~mL}$ 即得人工肠液(中国药典 2005 版第 二部, 未加蛋白酶). 含有胃蛋白酶的模拟胃液和含有 胰蛋白酶的模拟肠液的配制参照中国药典 2005 版第二 部.

称取 $15.0 \mathrm{mg}$ 载药微球样品加入离心管中, 加入 6.0 $\mathrm{mL}$ 的 $\mathrm{pH}=1.2$ 的不含酶的模拟胃液, 在 $37{ }^{\circ} \mathrm{C}$ 振荡箱中 振荡，每隔 $15 \mathrm{~min}$ 离心，取 $0.5 \mathrm{~mL}$ 上清液备测，并向离 心管中补加 $0.5 \mathrm{~mL}$ 缓冲液. $2 \mathrm{~h}$ 后，换为 $\mathrm{pH}=6.8$ 的不含 酶的模拟肠液, 每隔 $20 \mathrm{~min}$ 取样一次. 重复以上过程测 定. 通过紫外分光光度计测定 $334 \mathrm{~nm}$ 处的吸收, 通过标 准曲线测得上清液中利福平浓度. 每个样品做 3 个平行 实验, 取平均值.

载药微球在含有胃蛋白酶的模拟胃液和含有胰蛋 白酶的模拟肠液中的释放与无酶的模拟胃液和模拟肠 液中的释放步骤类似.

4.4.4 载药微球在模拟消化道 $\mathrm{pH}$ 值的缓冲液中的形貌 变化

称取一定量的载药微球放入离心管中, 加入等体积 的 $\mathrm{pH}=1.2$ 的不含酶的模拟胃液, 放入 $37{ }^{\circ} \mathrm{C}$ 恒温振荡 箱中以 $100 \mathrm{r} / \mathrm{min}$ 速度振荡, 于 $1 \mathrm{~h}$ 后将离心管取出, 离 心, 倾去上清液, 用二次水洗涤并液氮速冻. 同样称取 一定量的载药微球放入离心管中, 加入等体积的 $\mathrm{pH}=$ 6.8 的不含酶的模拟肠液, 放入 $37{ }^{\circ} \mathrm{C}$ 恒温振荡箱中以 $100 \mathrm{r} / \mathrm{min}$ 速度振荡，于 $0.5 \mathrm{~h}$ 后将离心管取出，离心，倾 去上清液，用二次水洗涤并液氮速冻. 将得到的样品在 扫描电镜下观察表面形貌. 


\subsection{5 空白微球的细胞毒性分析}

通过 MTT 法以 SDS 作为阳性对照检测聚氨基酸微 球对 HeLa 细胞的毒性. 在 96 孔板中按照 7000 细胞/ 孔的密度种植 HeLa 细胞, 并在每个孔内加入 $200 \mu \mathrm{L}$ 的 DMEM 培养基. DMEM 培养基的主要组成为 $10 \%$ 牛血 清, $50 \mathrm{IU} \cdot \mathrm{mL}^{-1}$ 的青需素和 $50 \mathrm{IU} \cdot \mathrm{mL}^{-1}$ 的链需素. 将细 胞培养 $24 \mathrm{~h}$, 细胞培养的外部环境为 $37^{\circ} \mathrm{C}$, 二氧化碳 $\left(\mathrm{CO}_{2}\right)$ 的含量为 $5 \%(\mathrm{~V} / \mathrm{V})$. 当细胞培养 $24 \mathrm{~h}$ 后将培养液 移除并在每个孔中加入 $200 \mu \mathrm{L}$ 含空白 PLG 微球的新鲜 培养液, 空白 PLG 微球的浓度范围为 $0 \sim 0.1 \mathrm{mg} \cdot \mathrm{mL}^{-1}$. 将细胞与载体材料共培养 $72 \mathrm{~h}$ 后向每个孔中加入 20.0 $\mu \mathrm{L}$ MTT 原液(5.0 mg・mL ${ }^{-1}$, PBS 溶液). 将细胞继续培养 $4 \mathrm{~h}$ 后进行 MTT 测试. 仪器为 Bio-Rad 680 酶标仪, 吸光 度检测波长为 $490 \mathrm{~nm}$. 细胞存活率通过下列公式计算:

Cell Viability $(\%)=(A$ 样品 $/ A$ 对照 $) \times 100$

其中, $A$ 样品和 $A$ 对照分别表示样品组和空白组的吸光度.

\section{References}

[1] Alessandra, M.; Lucia, Z.; Maria, D. D. C.; Anastasia, F.; Andrea, G. Adv. Drug Delivery Rev. 2012, 64, 540.

[2] Lee, W. A.; Narog, B. A.; Patapoff, T. W.; Wang, Y. J. J. Pharm. Sci. 1991, 80,725.

[3] Brain, J. D. Diabetes Technol. Ther. 2007, 9, S4.

[4] Alabraba, V.; Farnsworth, A.; Leigh, R.; Dodson, P.; Gough, S. C. L.; Smyth, T. Diabetes Technol. Ther. 2009, 11, 427.

[5] Chen, H.; Zhu, H.; Zheng, J.; Mou. D.; Wan, J.; Zhang, J.; Shi, T.; Zhao, Y.; Xu, H.; Yang, X. J. Controlled Release 2009, 139, 63.

[6] Thomas, B. J.; Finnin, B. C. Drug Discovery Today 2004, 9, 697.
[7] Molokhia, S. A.; Thomas, S. C.; Garff, K. J.; Mandell, K. J.; Wirostko, B. M. J. Ocul. Pharmacol. Th. 2013, 29, 92.

[8] Wang, D.; Xuan, H. X.; Lin, Q. S. Pharm. Biotechnol. 2000, 7, 57. (王乑，宣海星，林其谁，药物生物技术, 2000, 7, 57.)

[9] Iyer, H.; Khedkar, A.; Verma, M. Diabetes Obes. Metab. 2010, 12, 179.

[10] Mukhopadhyay, P.; Mishra, R.; Rana, D.; Kundu, P. P. Prog. Polym. Sci. 2012, 37, 1457.

[11] Yang, W. H.; Yu, S. Y.; Chen, S.; Liu, Y. Z.; Shao, Z. Z; Chen, X. Acta Chim. Sinica 2014, 72, 1164. (杨文华，俞淑英，陈胜，刘也 卓, 邵正中, 陈新, 化学学报, 2014, 72, 1164.)

[12] Jia, X. K.; Yin, J. J.; He, D. G.; He, X. X.; Wang, K. M.; Chen, M.; Li, Y. H. J. Biomed. Nanotechnol. 2013, 9, 2063.

[13] Osada, K.; Christie, R. J.; Kataoka, K. J. R. Soc. Interface 2009, 6 , S325.

[14] Deming, T. J. Adv. Drug Delivery Rev. 2002, 54, 1145

[15] Ding, J. X.; Xiao, C. S.; Tang, Z. H.; Zhuang, X. L.; Chen, X. S. Macromol. Biosci. 2011, 11, 192.

[16] Couffin-Hoarau, A. C.; Boustta, M.; Vert, M. J. Polym. Sci., Part A: Polym. Chem. 2001, 39, 3475.

[17] Kohn, J.; Langer, R. J. Am. Chem. Soc. 1987, 109, 817.

[18] Xu, X.; Wu, G. L.; Zhang, J.; Wang, Y. N.; Fan, Y. H.; Ma, J. B. Acta Chim. Sinica 2008, 66, 1102. (徐旭, 伍国琳，张洁，王亦农, 范云鸽, 马建标, 化学学报, 2008, 66, 1102.)

[19] Chen, L., Tian, H. Y.; Chen. J.; Chen, X. S.; Huang, Y. B.; Jing, X. B. J. Gene Med. 2010, 12, 64

[20] Li, C. Adv. Drug Delivery Rev. 2002, 54, 695.

[21] Zhao, L.; Ding, J. X.; Xiao, C. S.; He, P.; Tang, Z. H.; Pang, X.; Zhuang, X. L.; Chen, X. S. J. Mater. Chem. 2012, 22, 12319.

[22] He, N.; Sun, H. C.; Xu, H. X.; Shao, Z. Z. Chem. J. Chin. Univ. 2014，35，2019. (何宁, 孙贺春, 徐欢喜, 邵张章, 高等学校化学 学报, 2014, 35, 2019.)

[23] Zhang, Z.; Chen, L.; Deng, M. X.; Bai, Y. Y.; Chen, X. S.; Jing, X. B. J. Polym. Sci., Part A: Polym. Chem. 2011, 49, 2941.

[24] Han, J. D.; Ding, J. X.; Wang, Z. C.; Yan, S. F.; Zhuang, X. L.; Chen, X. S.; Yin, J. B. Sci. China, Chem. 2013, 56, 729.

[25] Leach, W. T.; Simpson, D. T.; Val, T. N.; Anuta, E. C.; Yu, Z. S.; Williams, R. O.; Johnston, K. P. J. Pharm. Sci. 2005, 94, 56.

[26] Sah, H. K. J. Pharm. Sci. 1997, 86, 1315.

(Cheng, F.) 